\title{
Sequential Proton Coupled Electron Transfer (PCET): Dynamics Observed over 8 Orders of Magnitude in Time.
}

\author{
Luke MacAleese* $^{\dagger}$, Sylvain Hermelin', Krystel El Hage ${ }^{\|}$, Pierre Chouzenoux ${ }^{\dagger}$, Alexander Kulesza ${ }^{\dagger}$, \\ Rodolphe Antoine ${ }^{\dagger}$, Luigi Bonacina', Markus Meuwly ${ }^{\|}$, Jean-Pierre Wolf', Philippe Dugourd ${ }^{\dagger}$ \\ ${ }^{\dagger}$ Institut lumière matière, UMR5306 Université Claude Bernard Lyon1-CNRS, Université de Lyon 69622 Villeurbanne \\ cedex, France \\ Group of Applied Physics (GAP) Biophotonics, Université de Genève, Chemin de Pinchat 22, CH-1211 Geneva, Switzer- \\ land \\ " Department of Chemistry, University of Basel, Klingelbergstr 80, CH-4056 Basel, Switzerland \\ KEYWORDS Pump-probe spectroscopy; action spectroscopy; photo-triggered charge transfer; electron and proton transfer; \\ tryptophan-containing peptide; metal-peptide complex
}

\section{SUPPORTING INFORMATION}

\section{Supplementary Tables}

Tables below list atomic charges used for simulations performed within this work (Table S1 and S2), and energy/coordinates of reactant, product and transition state on the optimized proton transfer pathway (Table S3).

Table S1. Tryptophan atomic charges*.

\begin{tabular}{|c|c|c|c|c|c|c|c|c|c|}
\hline $\operatorname{Trp}$ & $\mathrm{HF} / 6-31 \mathrm{G}(\mathrm{d})^{\mathrm{a}}$ & $\mathrm{CHARMM}^{\mathrm{b}}$ & CHARMM*c & Trp- & $\mathrm{HF} / 6-31 \mathrm{G}(\mathrm{d})^{\mathrm{a}}$ & CHARMM $^{* c}$ & Trp + & $\mathrm{HF} / 6-31 \mathrm{G}(\mathrm{d})^{\mathrm{a}}$ & CHARMM* \\
\hline $\mathrm{CB}$ & -0.39 & -0.18 & -0.24 & $\mathrm{CB}$ & -0.35 & -0.245 & $\mathrm{CB}$ & -0.46 & -0.26 \\
\hline HB1 & 0.12 & 0.09 & 0.12 & HB1 & 0.07 & 0.09 & HB1 & 0.19 & 0.17 \\
\hline $\mathrm{CG}$ & 0.09 & -0.03 & 0.06 & CG & -0.06 & -0.07 & $\mathrm{CG}$ & 0.24 & 0.28 \\
\hline CD1 & -0.22 & 0.04 & -0.12 & $\mathrm{CD} 1$ & 0.02 & -0.16 & $\mathrm{CD} 1$ & 0.2 & 0.2 \\
\hline NE1 & -0.45 & -0.61 & -0.6 & NE1 & -0.76 & -0.85 & NE1 & -0.49 & -0.64 \\
\hline HE1 & 0.4 & 0.38 & 0.4 & & & & HE1 & 0.45 & 0.57 \\
\hline CE2 & 0.23 & 0.13 & 0.13 & CE2 & 0.44 & 0.195 & CE2 & 0.38 & 0.38 \\
\hline $\mathrm{CD} 2$ & 0.05 & -0.02 & -0.05 & $\mathrm{CD} 2$ & -0.05 & -0.13 & $\mathrm{CD} 2$ & -0.11 & -0.11 \\
\hline CE3 & -0.18 & -0.12 & -0.18 & CE3 & -0.2 & -0.2 & CE3 & -0.04 & -0.13 \\
\hline HZ3 & 0.16 & 0.12 & 0.18 & $\mathrm{HZ3}$ & 0.13 & 0.2 & $\mathrm{HZ3}$ & 0.19 & 0.13 \\
\hline $\mathrm{CZ2}$ & -0.32 & -0.12 & -0.18 & $\mathrm{CZ2}$ & -0.32 & -0.2 & CZ2 & -0.29 & -0.13 \\
\hline $\mathrm{HZ2}$ & 0.18 & 0.12 & 0.18 & HZ2 & 0.14 & 0.2 & HZ2 & 0.22 & 0.13 \\
\hline $\mathrm{CH} 2$ & -0.07 & -0.12 & -0.18 & $\mathrm{CH} 2$ & -0.18 & -0.2 & $\mathrm{CH} 2$ & 0.03 & -0.13 \\
\hline $\mathrm{HH} 2$ & 0.14 & 0.12 & 0.18 & $\mathrm{HH} 2$ & 0.11 & 0.2 & $\mathrm{HH} 2$ & 0.16 & 0.13 \\
\hline
\end{tabular}

* All charges are given in units of e. ${ }^{a}$ from HF/6-31G(d) ESP fits, ${ }^{b}$ from CHARMM 22 parameter set and ${ }^{\mathrm{c}}$ the corresponding adjusted ESP CHARMM* charges. 
Table S2. Histidine atomic charges*.

\begin{tabular}{llll}
\hline His & HF/6-31G(d) & CHARMM $^{\mathrm{b}}$ & CHARMM $^{*}$ \\
\hline CB & -0.39 & -0.09 & -0.16 \\
HB1 & 0.15 & 0.09 & 0.14 \\
HB2 & 0.13 & 0.09 & 0.14 \\
ND1 & -0.41 & -0.36 & -0.41 \\
HD1 & 0.36 & 0.32 & 0.33 \\
CG & 0.20 & -0.05 & -0.04 \\
CE1 & 0.23 & 0.25 & 0.24 \\
HE1 & 0.12 & 0.13 & 0.13 \\
NE2 & -0.51 & -0.70 & -0.71 \\
CD2 & -0.03 & 0.22 & 0.20 \\
HD2 & 0.15 & 0.10 & 0.15
\end{tabular}

\begin{tabular}{llll}
\hline His $^{+}$ & HF/6-31G(d) & CHARMM & CHARMM* \\
\hline CB & -0.39 & -0.05 & -0.20 \\
HB1 & 0.16 & 0.09 & 0.16 \\
HB2 & 0.18 & 0.09 & 0.16 \\
ND1 & -0.25 & -0.51 & -0.35 \\
HD1 & 0.41 & 0.44 & 0.41 \\
CE1 & 0.08 & 0.32 & 0.16 \\
HE1 & 0.26 & 0.18 & 0.26 \\
CG & 0.25 & 0.19 & 0.25 \\
NE2 & -0.19 & -0.51 & -0.33 \\
HE2 & 0.41 & 0.44 & 0.41 \\
CD2 & -0.20 & 0.19 & -0.10 \\
HD2 & 0.27 & 0.13 & 0.17 \\
\hline
\end{tabular}

* All charges are given in units of e. ${ }^{a}$ from HF/6-31G(d) ESP fits, ${ }^{b}$ from CHARMM 22 parameter set and ${ }^{c}$ the corresponding adjusted ESP CHARMM* charges.

Table S3. Reactant, Product and Transition State structures coordinates.

\begin{tabular}{rrrrr} 
& & \multicolumn{3}{c}{ Transition state } \\
\cline { 3 - 5 } & & (1 imaginary & freq. $:-729.8099 \mathbf{~ c m}^{-1}$ ) \\
1 & $\mathrm{C}$ & E(UB3LYP) $=-1915.33265$ a.u. \\
\cline { 3 - 5 } 2 & $\mathrm{C}$ & -1.505079 & -0.889602 & -0.62265 \\
3 & $\mathrm{~N}$ & -2.813515 & -1.147606 & -0.940932 \\
4 & $\mathrm{C}$ & -1.784686 & -2.516251 & -0.813682 \\
5 & $\mathrm{~N}$ & -0.874537 & -2.081488 & -0.317675 \\
6 & $\mathrm{C}$ & -3.963265 & -0.278094 & -1.317707 \\
7 & $\mathrm{C}$ & -5.109319 & -0.294437 & -0.234222 \\
8 & $\mathrm{~N}$ & -6.390631 & -0.674472 & -0.781346 \\
9 & $\mathrm{C}$ & -6.631176 & -1.946686 & -1.180415 \\
10 & $\mathrm{C}$ & -8.0191 & -2.247258 & -1.701991 \\
11 & $\mathrm{C}$ & -5.223775 & 1.109345 & 0.368054 \\
12 & $\mathrm{O}$ & -6.076965 & 1.905219 & -0.008233 \\
13 & $\mathrm{~N}$ & -4.247761 & 1.386295 & 1.267563 \\
14 & $\mathrm{C}$ & -3.840718 & 2.733557 & 1.615402 \\
15 & $\mathrm{C}$ & -2.308854 & 2.719522 & 1.698552 \\
16 & $\mathrm{O}$ & -1.709662 & 1.691996 & 2.004926 \\
17 & $\mathrm{~N}$ & -1.676911 & 3.900639 & 1.467413 \\
18 & $\mathrm{C}$ & -0.234482 & 3.929769 & 1.382187 \\
19 & $\mathrm{C}$ & 0.296248 & 3.287902 & 0.082087 \\
20 & $\mathrm{O}$ & -0.442643 & 2.861297 & -0.796054 \\
21 & $\mathrm{~N}$ & 1.658679 & 3.285877 & -0.003486 \\
22 & $\mathrm{C}$ & 2.353791 & 2.619091 & -1.080867 \\
23 & $\mathrm{C}$ & 3.401633 & 1.655128 & -0.515754 \\
24 & $\mathrm{O}$ & 3.547617 & 1.488933 & 0.690574 \\
25 & $\mathrm{~N}$ & 4.148113 & 0.996727 & -1.451696 \\
26 & $\mathrm{C}$ & 5.314674 & 0.226268 & -1.071464 \\
27 & $\mathrm{C}$ & 6.421159 & 0.545655 & -2.098869 \\
28 & $\mathrm{O}$ & 6.125112 & 0.950006 & -3.215081 \\
29 & $\mathrm{C}$ & 5.050123 & -1.32154 & -1.090532 \\
30 & $\mathrm{C}$ & 3.861793 & -1.719311 & -0.286207 \\
31 & $\mathrm{C}$ & 3.770894 & -2.100592 & 1.085274 \\
32 & $\mathrm{C}$ & 2.391226 & -2.325403 & 1.354218
\end{tabular}

\begin{tabular}{rrr}
\multicolumn{3}{c}{ Reactant } \\
\hline & & \\
\hline E(UB3LYP) $=-1915.35625$ a.u. \\
\hline-1.669591 & -1.322998 & -0.823885 \\
-3.028293 & -1.416259 & -0.99791 \\
-3.336446 & -2.734761 & -0.728565 \\
-2.189792 & -3.378926 & -0.416809 \\
-1.152934 & -2.553562 & -0.466936 \\
-4.086979 & -0.426702 & -1.352337 \\
-5.081305 & -0.149549 & -0.162862 \\
-6.460084 & -0.408377 & -0.509158 \\
-6.915732 & -1.668549 & -0.712641 \\
-8.394591 & -1.817131 & -0.998899 \\
-4.97596 & 1.31426 & 0.291246 \\
-5.87874 & 2.122946 & 0.10164 \\
-3.791023 & 1.624317 & 0.868788 \\
-3.404895 & 2.975174 & 1.207868 \\
-1.881106 & 2.99992 & 1.355454 \\
-1.238797 & 1.948811 & 1.453678 \\
-1.304189 & 4.21991 & 1.40171 \\
0.113998 & 4.411087 & 1.705512 \\
1.066395 & 4.214999 & 0.509655 \\
1.758945 & 5.130671 & 0.089219 \\
1.07429 & 2.949649 & -0.006931 \\
2.019386 & 2.569841 & -1.027756 \\
2.902922 & 1.408771 & -0.560618 \\
2.55914 & 0.645818 & 0.344564 \\
4.065353 & 1.245699 & -1.24078 \\
5.082264 & 0.296447 & -0.851494 \\
6.390169 & 0.779749 & -1.521403 \\
6.349544 & 1.504411 & -2.504585 \\
4.79174 & -1.178887 & -1.317458 \\
3.66836 & -1.831142 & -0.590296 \\
3.697554 & -2.520971 & 0.66002 \\
2.368476 & -2.942721 & 0.932797
\end{tabular}

\begin{tabular}{rrr}
\multicolumn{3}{c}{ Product } \\
\hline & & \\
\hline E(UB3LYP) $=$ & -1915.33655 a.u. \\
\hline-0.972773 & -1.601058 & -0.407487 \\
-2.331784 & -1.738343 & -0.528097 \\
-2.594888 & -3.093796 & -0.425885 \\
-1.447207 & -3.75569 & -0.261114 \\
-0.449743 & -2.869597 & -0.250224 \\
-3.399561 & -0.724164 & -0.793409 \\
-4.64633 & -0.777176 & 0.165449 \\
-5.835264 & -1.333919 & -0.450061 \\
-6.074046 & -2.650596 & -0.63554 \\
-7.440838 & -3.020805 & -1.165278 \\
-5.013156 & 0.669789 & 0.564584 \\
-5.973509 & 1.239327 & 0.058609 \\
-4.165369 & 1.23996 & 1.454358 \\
-4.067639 & 2.678088 & 1.624598 \\
-2.586198 & 3.05546 & 1.496546 \\
-1.715651 & 2.198932 & 1.693254 \\
-2.302842 & 4.336794 & 1.190539 \\
-0.934191 & 4.858713 & 1.165566 \\
-0.139023 & 4.544707 & -0.120178 \\
0.276866 & 5.438815 & -0.842605 \\
0.075789 & 3.215987 & -0.343412 \\
0.902079 & 2.75022 & -1.442182 \\
1.862654 & 1.682548 & -0.914563 \\
1.434198 & 0.699418 & -0.299482 \\
3.17613 & 1.891864 & -1.154453 \\
4.250839 & 1.115403 & -0.564586 \\
5.465923 & 2.067172 & -0.498496 \\
5.52918 & 3.055312 & -1.215102 \\
4.634246 & -0.181791 & -1.382976 \\
3.994176 & -1.410299 & -0.833622 \\
4.494545 & -2.28464 & 0.178533 \\
3.437473 & -3.200273 & 0.458746
\end{tabular}




\begin{tabular}{|c|c|c|c|c|c|c|c|c|c|c|}
\hline 33 & $\mathrm{~N}$ & 1.656557 & -2.099245 & 0.171976 & 1.557396 & -2.537171 & -0.141751 & 2.313873 & -2.917437 & -0.35446 \\
\hline 34 & C & 2.509747 & -1.746219 & -0.773364 & 2.302284 & -1.888415 & -1.022475 & 2.660683 & -1.890783 & -1.10293 \\
\hline 35 & C & 4.712751 & -2.274828 & 2.113196 & 4.717089 & -2.830934 & 1.577046 & 5.716008 & -2.390507 & 0.862417 \\
\hline 36 & C & 4.260388 & -2.660458 & 3.380438 & 4.384844 & -3.534133 & 2.73734 & 5.862442 & -3.400338 & 1.8199 \\
\hline 37 & C & 2.899807 & -2.86781 & 3.620164 & 3.06393 & -3.926324 & 2.984151 & 4.813703 & -4.286414 & 2.086239 \\
\hline 38 & C & 1.935334 & -2.70167 & 2.599317 & 2.025722 & -3.636378 & 2.075347 & 3.581486 & -4.196456 & 1.402957 \\
\hline 39 & 0 & -5.760055 & -2.827007 & -1.115223 & -6.16418 & -2.65234 & -0.666831 & -5.232741 & -3.52986 & -0.383777 \\
\hline 40 & $\mathrm{H}$ & -7.937731 & -2.676444 & -2.705064 & -8.926468 & -0.862831 & -1.038802 & -8.073351 & -2.155412 & -1.378116 \\
\hline 41 & $\mathrm{H}$ & -8.668007 & -1.368123 & -1.737328 & -8.842684 & -2.443601 & -0.221225 & -7.940787 & -3.65574 & -0.426935 \\
\hline 42 & H & -8.480063 & -3.004961 & -1.060496 & -8.521787 & -2.340794 & -1.951082 & -7.320401 & -3.615093 & -2.075805 \\
\hline 43 & H & -7.078562 & 0.066962 & -0.862416 & -7.068547 & 0.40403 & -0.523671 & -6.550267 & -0.638444 & -0.650281 \\
\hline 44 & $\mathrm{H}$ & -4.84263 & -1.014684 & 0.546751 & -4.80457 & -0.801259 & 0.67426 & -4.395568 & -1.358277 & 1.060643 \\
\hline 45 & $\mathrm{H}$ & -3.88261 & -2.966157 & -0.931453 & -4.306761 & -3.066827 & -0.705812 & -3.575491 & -3.458713 & -0.4172 \\
\hline 46 & $\mathrm{H}$ & -3.598155 & 0.740313 & -1.478473 & -3.609092 & 0.502773 & -1.675755 & -2.924491 & 0.259415 & -0.730323 \\
\hline 47 & $\mathrm{H}$ & -4.394038 & -0.612414 & -2.266528 & -4.674648 & -0.789641 & -2.201079 & -3.770314 & -0.824772 & -1.820228 \\
\hline 48 & $\mathrm{H}$ & -0.994556 & 0.062962 & -0.581553 & -1.04051 & -0.448557 & -0.918967 & -0.337605 & -0.722978 & -0.410548 \\
\hline 49 & $\mathrm{H}$ & -1.617083 & -4.09712 & -0.273353 & -2.148774 & -4.429729 & -0.167681 & -1.346014 & -4.824592 & -0.153472 \\
\hline 50 & $\mathrm{H}$ & -3.540466 & 0.688837 & 1.481029 & -3.063029 & 0.928558 & 1.000061 & -3.321112 & 0.747946 & 1.732414 \\
\hline 51 & H & -4.244229 & 3.041021 & 2.590303 & -3.860625 & 3.299876 & 2.154596 & -4.428721 & 2.986185 & 2.615075 \\
\hline 52 & H & -4.223685 & 3.432646 & 0.86565 & -3.749438 & 3.671439 & 0.434455 & -4.697031 & 3.166625 & 0.875297 \\
\hline 53 & $\mathrm{H}$ & -2.19051 & 4.631592 & 0.993756 & -1.87209 & 5.04185 & 1.244884 & -3.057919 & 4.96925 & 0.96085 \\
\hline 54 & H & 0.176392 & 3.389823 & 2.240925 & 0.387787 & 3.716359 & 2.507003 & -0.401 & 4.454961 & 2.032753 \\
\hline 55 & H & 0.104954 & 4.968462 & 1.444027 & 0.25587 & 5.433001 & 2.057007 & -0.979702 & 5.944301 & 1.249834 \\
\hline 56 & H & 2.195752 & 3.403396 & 0.848236 & 0.55166 & 2.226237 & 0.480745 & -0.265972 & 2.540041 & 0.335872 \\
\hline 57 & H & 1.612104 & 2.082361 & -1.681801 & 1.508618 & 2.259276 & -1.952415 & 0.292571 & 2.29507 & -2.236365 \\
\hline 58 & H & 2.846304 & 3.342473 & -1.746495 & 2.616536 & 3.454883 & -1.266104 & 1.410799 & 3.61896 & -1.865886 \\
\hline 59 & H & 4.1607 & 1.308854 & -2.420311 & 4.355547 & 1.889697 & -1.973512 & 3.496152 & 2.750549 & -1.59773 \\
\hline 60 & H & 5.59904 & 0.523033 & -0.057286 & 5.173606 & 0.296619 & 0.241214 & 3.950081 & 0.815457 & 0.44502 \\
\hline 61 & $\mathrm{H}$ & 5.949552 & -1.830543 & -0.727206 & 5.709636 & -1.758714 & -1.167351 & 5.722913 & -0.298407 & -1.365096 \\
\hline 62 & $\mathrm{H}$ & 4.905088 & -1.620596 & -2.135385 & 4.595329 & -1.151791 & -2.394367 & 4.343339 & -0.012476 & -2.424627 \\
\hline 63 & $\mathrm{H}$ & 2.175038 & -1.5209 & -1.778822 & 1.876788 & -1.484135 & -1.931774 & 1.974857 & -1.4559 & -1.820451 \\
\hline 64 & H & 0.455628 & -2.151247 & -0.006205 & 0.489886 & -2.657569 & -0.226123 & 0.589135 & -3.073177 & -0.204988 \\
\hline 65 & $\mathrm{H}$ & 5.772448 & -2.117259 & 1.934586 & 5.746276 & -2.53835 & 1.390416 & 6.541149 & -1.715635 & 0.646757 \\
\hline 66 & $\mathrm{H}$ & 0.880433 & -2.861243 & 2.798158 & 1.004824 & -3.946194 & 2.273007 & 2.777144 & -4.894603 & 1.614114 \\
\hline 67 & $\mathrm{H}$ & 4.97367 & -2.799682 & 4.186121 & 5.158789 & -3.782102 & 3.456235 & 6.800317 & -3.502927 & 2.356563 \\
\hline 68 & $\mathrm{H}$ & 2.570191 & -3.164713 & 4.610914 & 2.829877 & -4.47138 & 3.893227 & 4.949418 & -5.06522 & 2.830687 \\
\hline 69 & $\mathrm{~N}$ & 7.691908 & 0.281216 & -1.704098 & 7.540993 & 0.296569 & -0.986694 & 6.454655 & 1.689266 & 0.354339 \\
\hline 70 & $\mathrm{H}$ & 8.441842 & 0.505795 & -2.34603 & 8.411975 & 0.634619 & -1.377222 & 7.245819 & 2.312015 & 0.458328 \\
\hline 71 & $\mathrm{H}$ & 7.933845 & 0.130046 & -0.735607 & 7.573899 & -0.105562 & -0.061008 & 6.297836 & 1.005588 & 1.081014 \\
\hline
\end{tabular}




\section{Supplementary Figures}
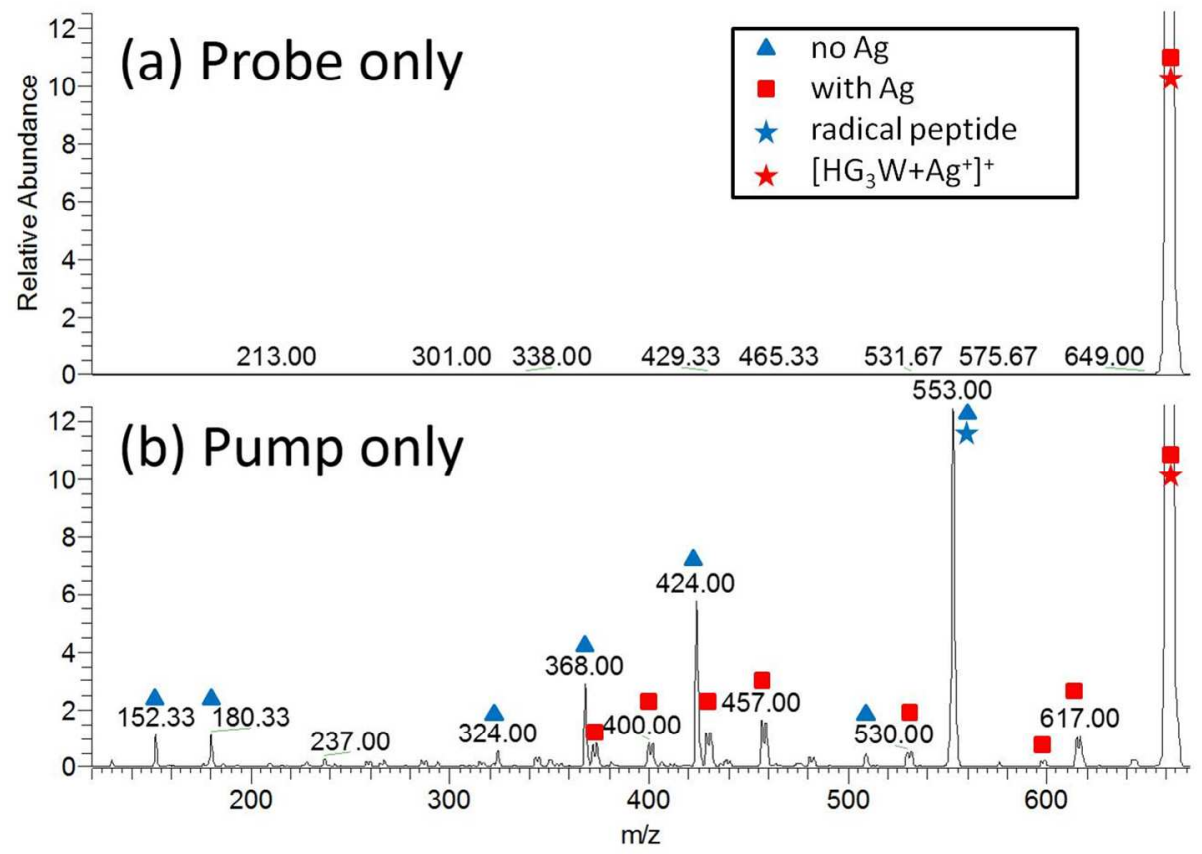

Figure S1. Typical photo-fragmentation mass spectra of $\left[\mathrm{HG}_{3} \mathrm{~W}+\mathrm{Ag}\right]^{+}$after a single pulse of either probe laser at $580 \mathrm{~nm}$ (a) or pump laser at $266 \mathrm{~nm}$ (b). Both laser pulse energies are identical to the one used for pump-probe experiments. Relative abundances (in \%) are normalized to the metal complex intensity in each spectrum. Pump beam generates mostly Ag-free fragments (blue triangle) and to a lesser extent silver containing fragments (red square). Both ion families are readily identified based on the silver-specific isotopic pattern. Probe has comparatively no effect. 


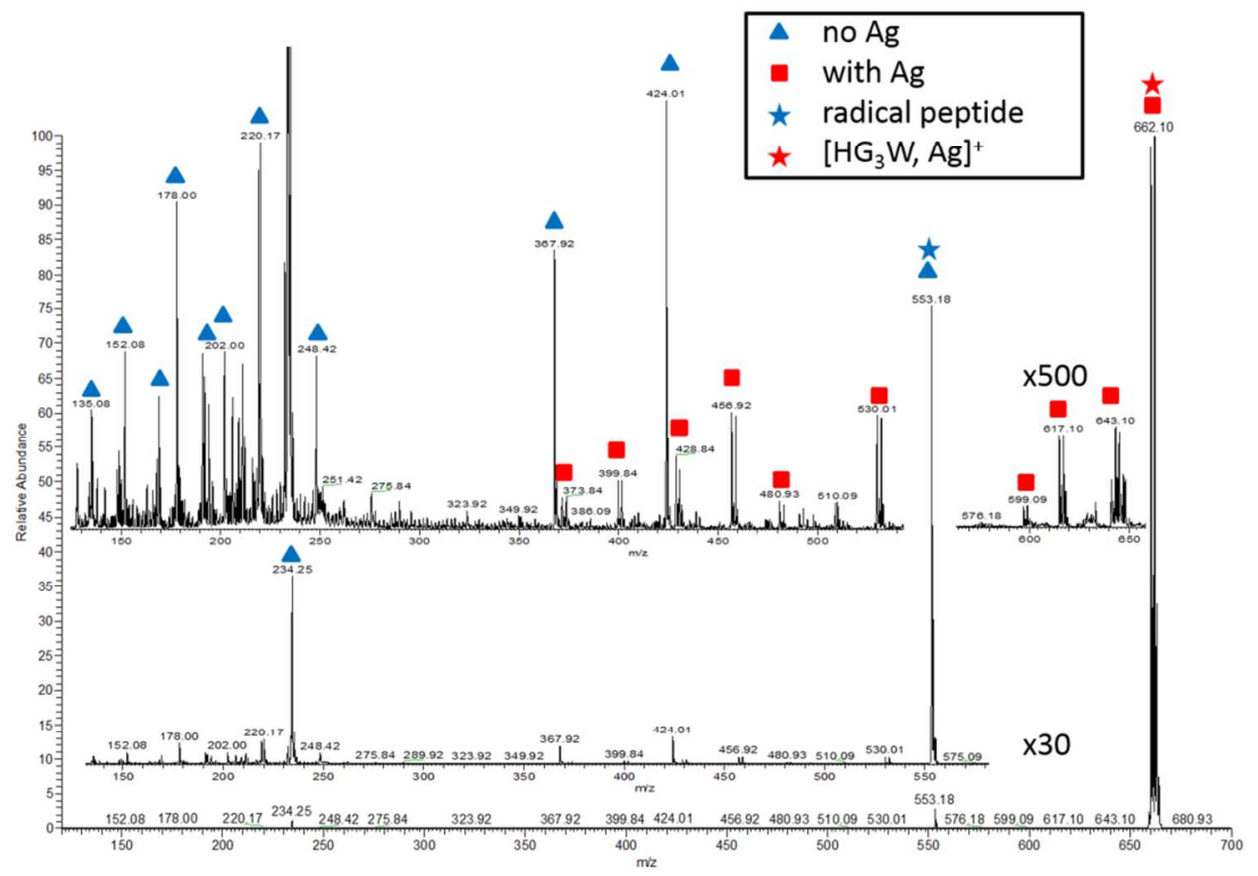

Figure S2. Typical mass spectrum after photo-fragmentation of $\left[\mathrm{HG}_{3} \mathrm{~W}+\mathrm{Ag}\right]^{+}$after pump and probe irradiation (mass spectra obtained for different pump-probe delays were averaged). Parent silver complex dissociates into silver-containing (red square) and silver-free (blue triangle) ions. Both ion families are readily identified based on the silver-specific isotopic pattern. 


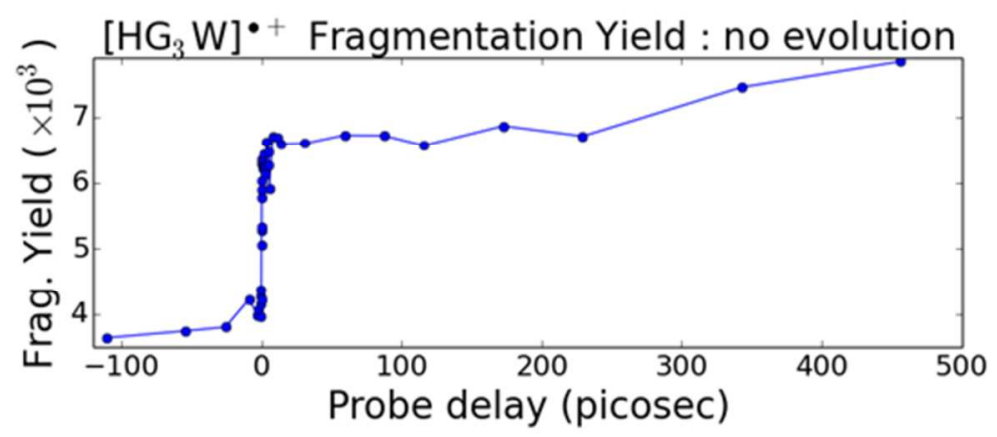

Figure S3. Evolution of radical peptide $\left[\mathrm{HG}_{3} \mathrm{~W}\right]^{++} \mathrm{FY}$ as a function of the pump-probe delay. Fragmentation yield of radical peptide exhibits a step-like behavior within the boundaries of the ultrafast experiment $(<500 \mathrm{ps})$. Prior to pump, the peptide is not radical and does not absorb the probe laser. After pump and charge transfer, a radical is formed on tryptophan and displays higher absorption properties. FY is constant after radical formation, which indicates that the timescale is too short to observe proton transfer. 


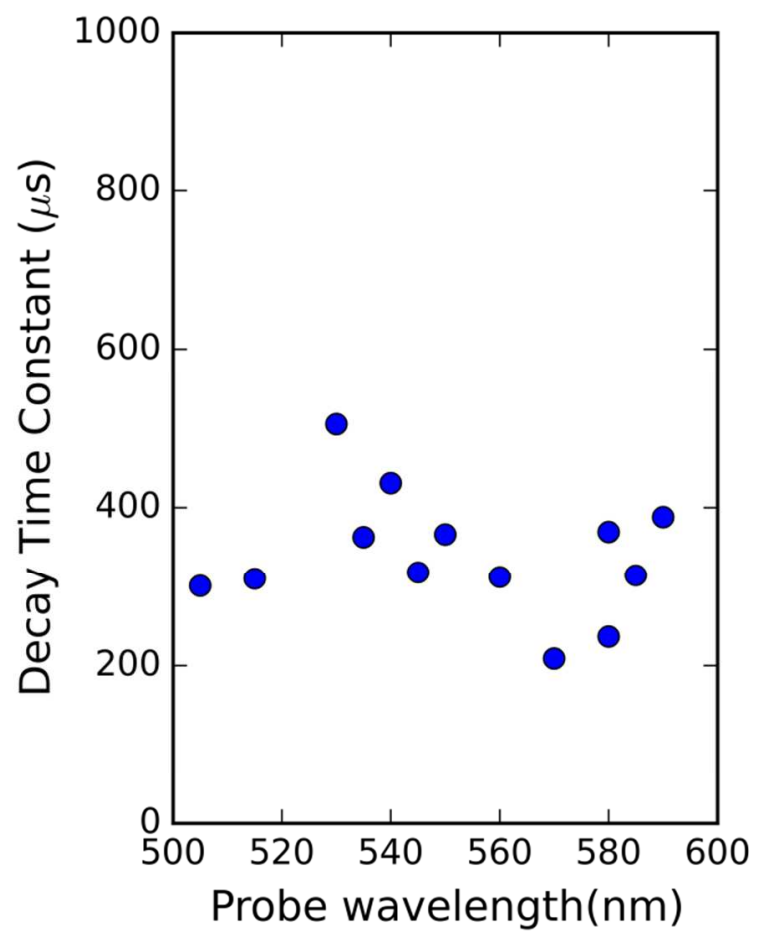

Figure S4. Time constant for proton transfer in $\left[\mathrm{HG}_{3} \mathrm{~W}\right]^{\cdot+}$ evaluated at different probe wavelengths. 


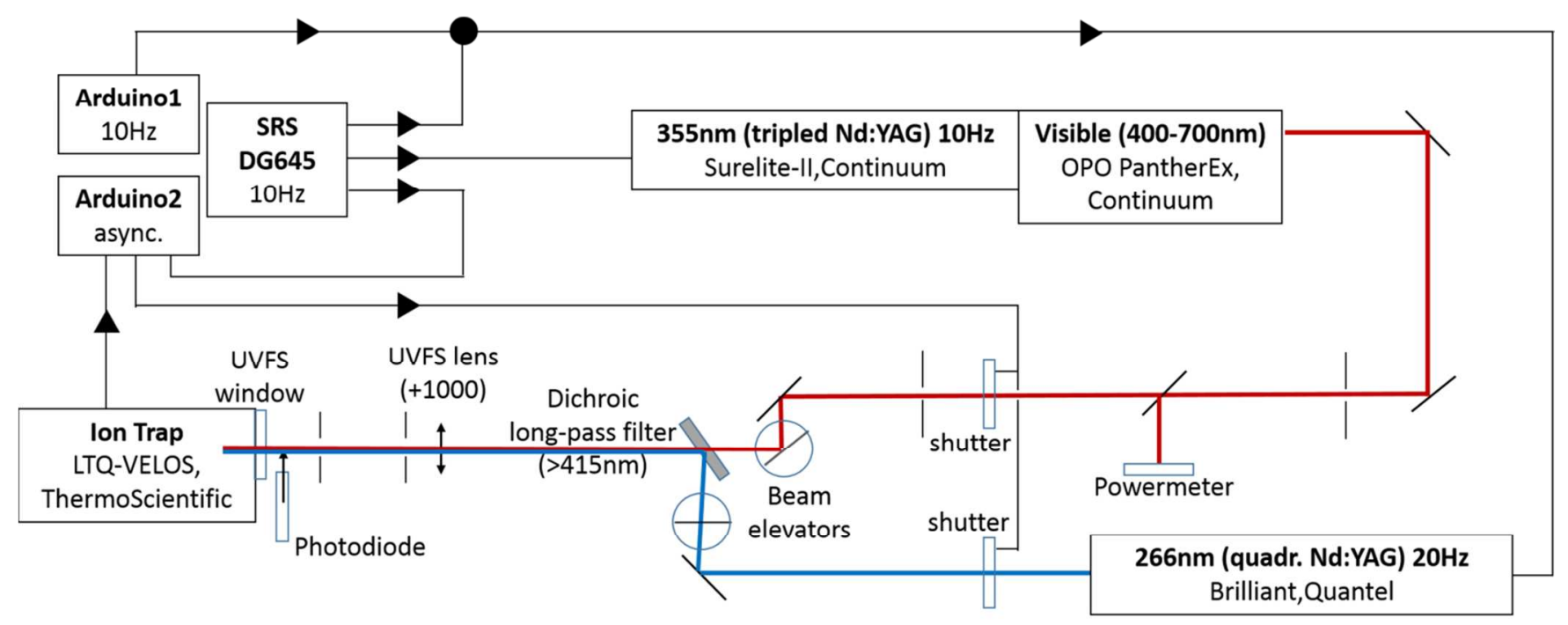

Figure S5. Setup for the nanosecond pump-probe delay experiments. 


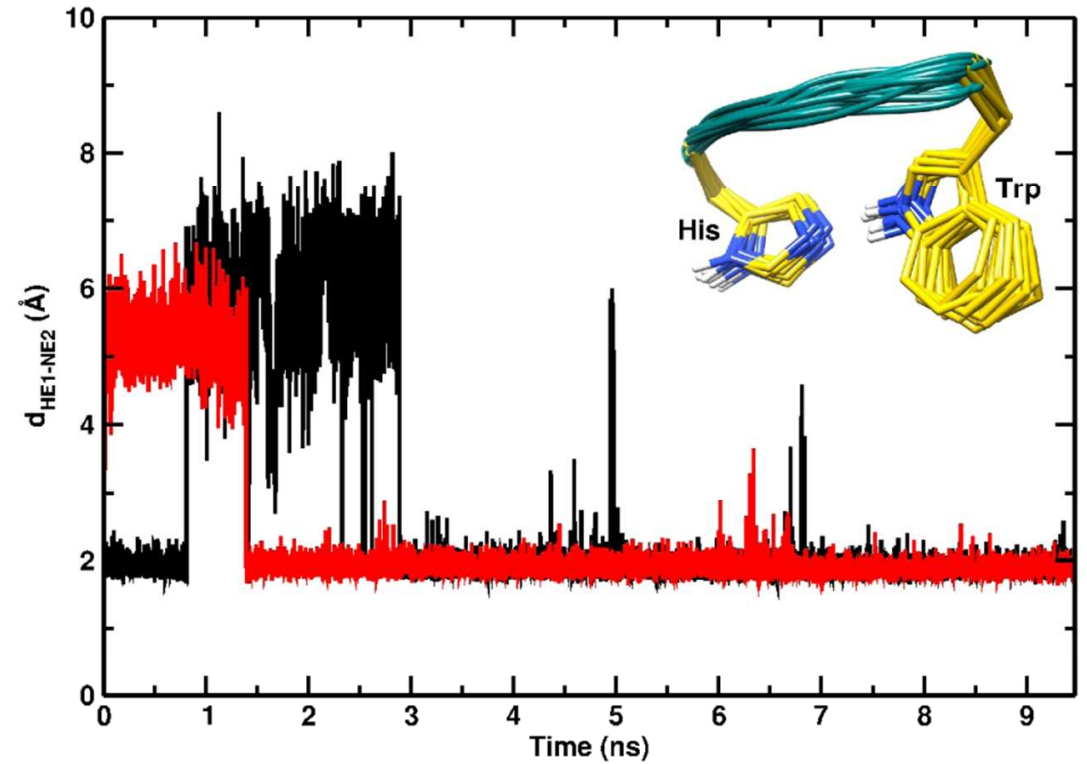

Figure S6 - Time history of the indoleNH-imidazoleN distance (HE1Trp-NE2His) distance in $\left[\mathrm{HG}_{3} \mathrm{~W}\right]^{\circ+}$ for two $10 \mathrm{~ns} \mathrm{MD}$ simulations starting from an extended peptide structure. The inset shows a superposition of instantaneous configurations. 
A

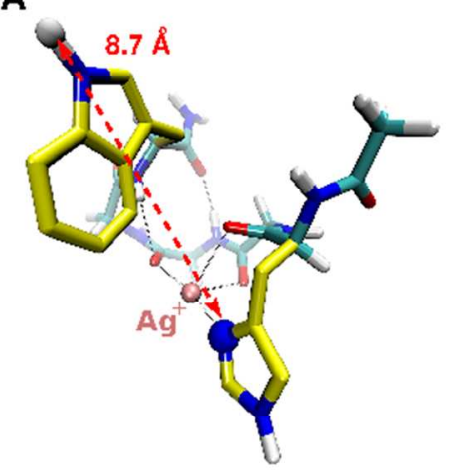

D

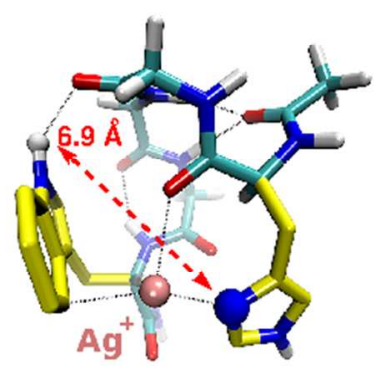

B

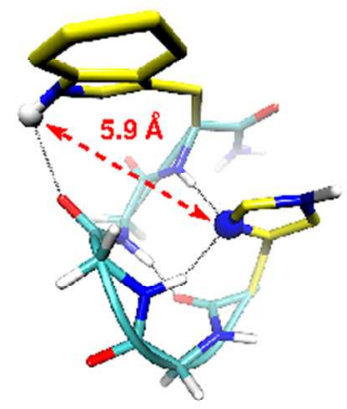

$\mathbf{E}$

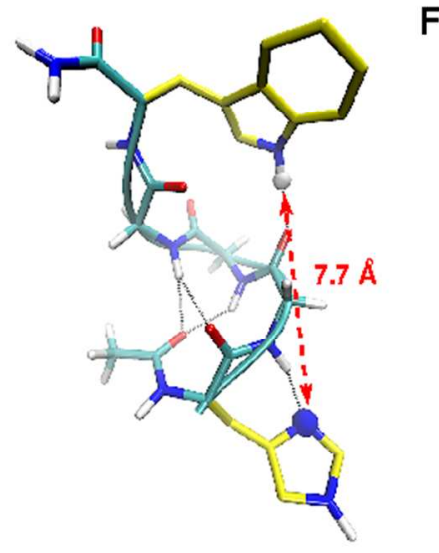

C

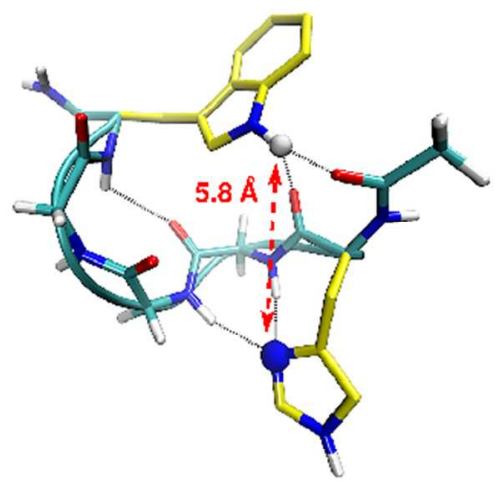

$\mathbf{F}$

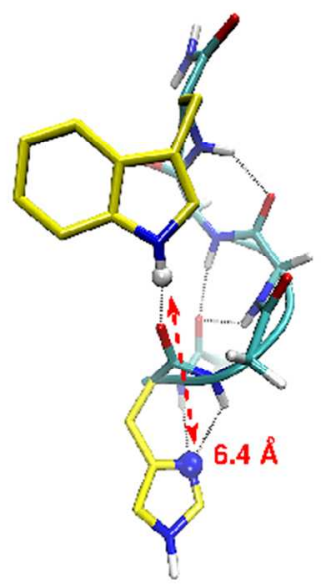

Figure S7 - Structures of $\mathrm{HG}_{3} \mathrm{~W}$ peptide. (A, D) Lowest energy structures obtained for the $\left[\mathrm{Ag}+\mathrm{HG}_{3} \mathrm{~W}\right]^{+}$complex (ref33 in MS). Typical hindered structures at equilibrium obtained for $\mathrm{HG}_{3} \mathrm{~W}^{*+}$ after silver has been removed from structure $\mathrm{A}(\mathrm{B}, \mathrm{C})$ or from structure $\mathrm{D}(\mathrm{E}, \mathrm{F})$. Dashed red lines indicate the indoleNH to imidazoleN distances. $\mathrm{H}$ atom on indoleN and $\mathrm{N}$ atom on imidazole are highlighted by vdW representations. 


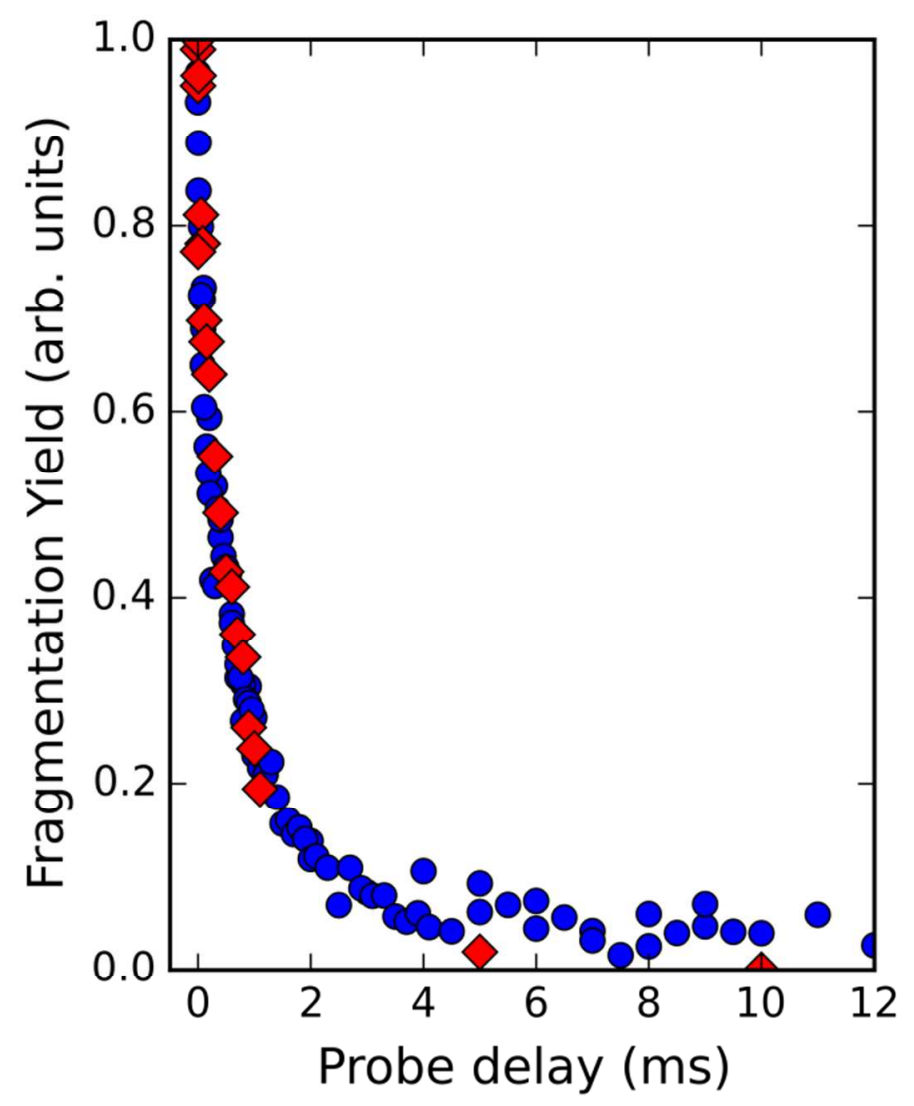

Figure S8. Evolution of $\left[\mathrm{HG}_{3} \mathrm{~W}\right]^{\circ+}$ radical peptide fragmentation yield as a function of the pump-probe delay at probe wavelength $550 \mathrm{~nm}$ for the deuterated (blue circles) and the non-deuterated species (red diamond). No difference for the experimental decay rate was found for the deuterated species compared to the fully hydrogenated system which yields a primary kinetic isotope effect (KIE) close to 1. 


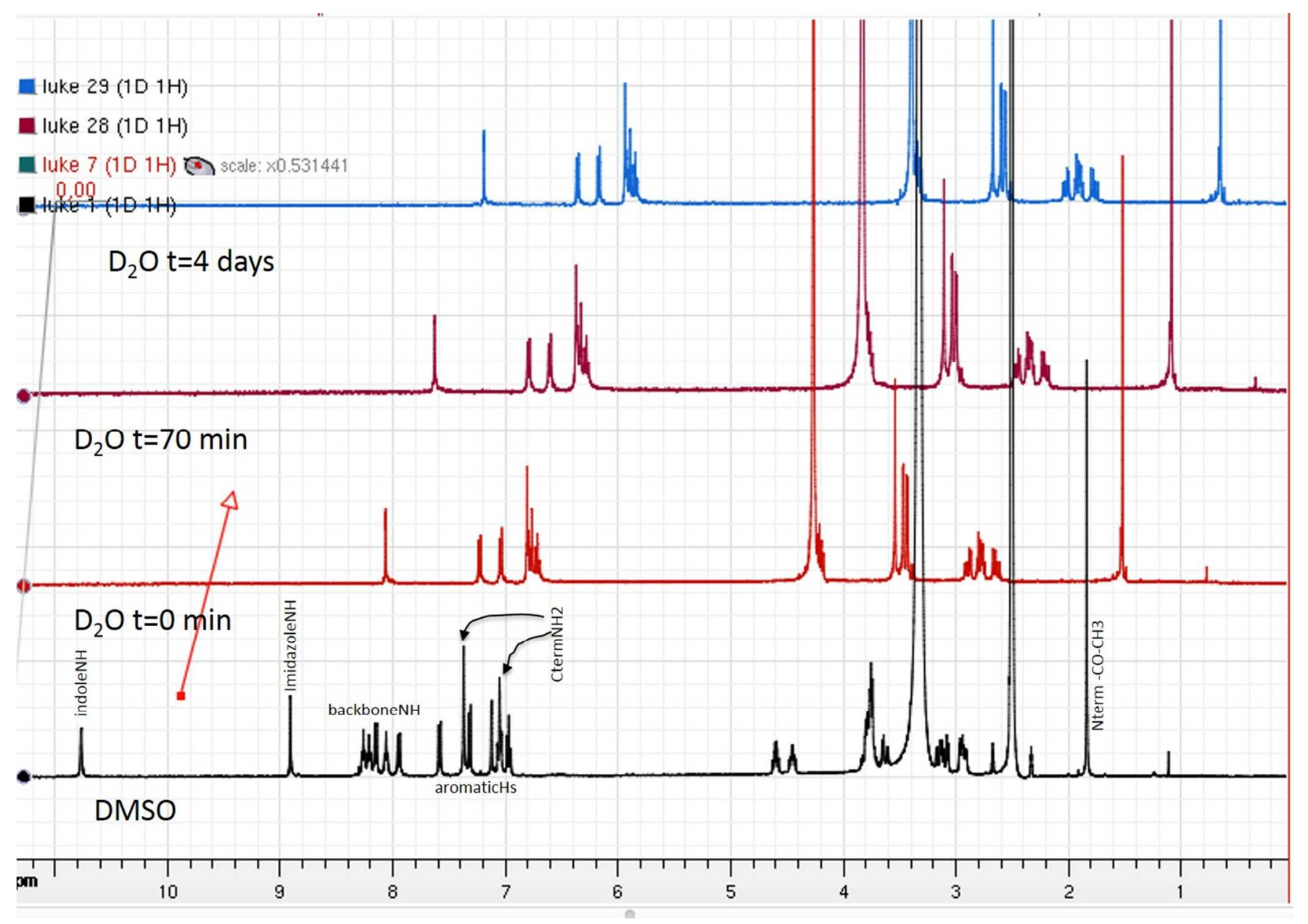

Figure S9. NMR ${ }^{1} \mathrm{H}$ spectra of $\mathrm{HG}_{3} \mathrm{~W}$ in DMSO (black) and in $\mathrm{D}_{2} \mathrm{O}$ immediately after dissolution (red), and after 70 minutes (dark red) and 4 days (blue). The indoleNH is immediately exchanged with a deuterium together with all backbone NHs and the Cterm NH2. 


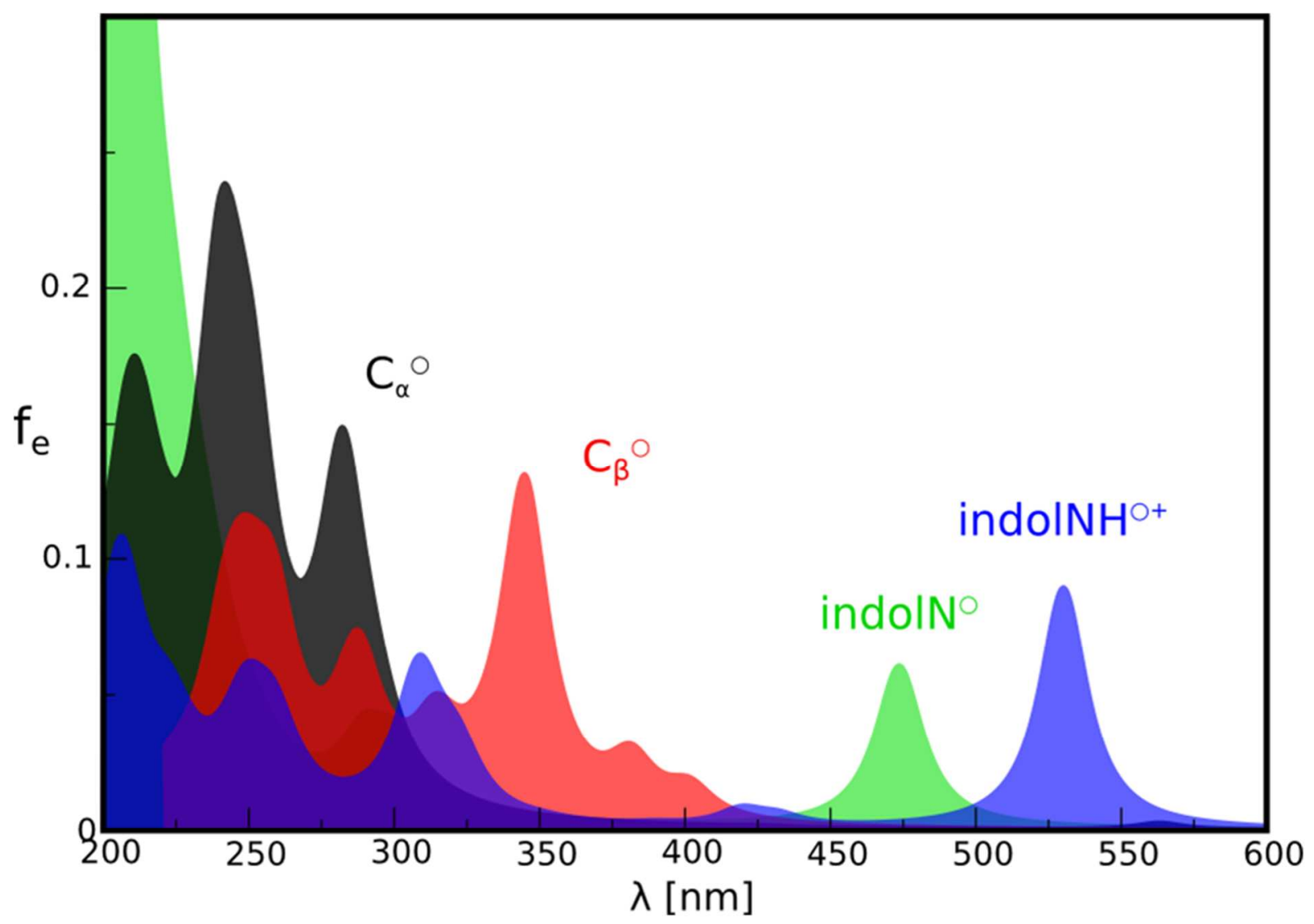

Figure S10. Absorption spectra $\mathrm{f}$ (oscillator strength $f_{e}$ ) from TDDFT calculations for Ac-W-NH $\mathrm{N}_{2}$ model radicals representing the initial and potential final states of the $\mathrm{HG}_{3} \mathrm{~W}^{*+}$ radical: indolNH ${ }^{*+}$ immediately after $\mathrm{Ag}$ release, and with protons translocated from $\mathrm{C}_{\alpha}, \mathrm{C}_{\beta}$ and indoleN $\left(\mathrm{C}_{\alpha}{ }^{\bullet}\right.$ : black, $\mathrm{C}_{\beta}{ }^{\bullet}$ : red, indolN ${ }^{\bullet}$ : green, respectively). Absorption lines are convoluted by Lorentzian functions with half-widths of 20 $\mathrm{nm}$. Intense transitions are found in the visible range for indolNH ${ }^{\circ+}(490-530 \mathrm{~nm})$ and indolN $(440-470 \mathrm{~nm})$ which matches the steadystate spectra (Fig. 3 and ref 34 in MS). This shows that the structure after proton transfer contains an indole radical as opposed to a radical on a carbon sites $\left(\mathrm{C}_{\alpha}\right.$ or $\left.\mathrm{C}_{\beta}\right)$. 